\title{
Automated biological target volume delineation for radiotherapy treatment planning using FDG-PET/CT
}

Maximilian Niyazi ${ }^{*}$, Sonja Landrock², Andreas Elsner ${ }^{3}$, Farkhad Manapov', Marcus Hacker², Claus Belka ${ }^{1}$ and Ute Ganswindt ${ }^{1}$

\begin{abstract}
Background: This study compared manually delineated gross tumour volume (GTV) and automatically generated biological tumour volume (BTV) based on fluoro-deoxy-glucose (FDG) positron emission tomography (PET)/CT to assess the robustness of predefined PET algorithms for radiotherapy (RT) planning in routine clinical practice.

Methods: RT-planning data from 20 consecutive patients (lung- (40\%), oesophageal- (25\%), gynaecological- (25\%) and colorectal (10\%) cancer) who had undergone FDG-PET/CT planning between 08/2010 and 09/2011 were retrospectively analysed, five of them underwent neoadjuvant chemotherapy before radiotherapy. In addition to manual GTV contouring, automated segmentation algorithms were applied-among these 38\%, 42\%, 47\% and 50\% SUV $_{\max }$ as well as the PERCIST total lesion glycolysis (TLG) algorithm. Different ratios were calculated to assess the overlap of GTV and BTV including the conformity index and the ratio GTV included within the BTV.

Results: Median age of the patients was 66 years and median tumour SUV $\max$ 9.2. Median size of the GTVs defined by the radiation oncologist was $43.7 \mathrm{ml}$. Median conformity indices were between 30.0-37.8\%. The highest amount of BTV within GTV was seen with the 38\% SUV $\max$ algorithm (49.0\%), the lowest with 50\% SUV $\max (36.0 \%)$. Best agreement was obtained for oesophageal cancer patients with a conformity index of $56.4 \%$ and BTV within GTV ratio of $71.1 \%$.

Conclusions: At present there is only low concordance between manually derived GTVs and automatically segmented FDG-PET/CT based BTVs indicating the need for further research in order to achieve higher volumetric conformity and therefore to get access to the full potential of FDG-PET/CT for optimization of radiotherapy planning.
\end{abstract}

Keywords: PET/CT, Planning study, BTV, Target delineation

\section{Introduction}

Over the recent years $\left[{ }^{18} \mathrm{~F}\right]$ FDG-PET imaging has become a valuable tool in oncology. Based on the higher sensitivity and specificity compared to conventional imaging modalities such as CT or MRI alone, PET is mainly employed for staging.

In addition, PET-CT may also be used as prognostic and/or predictive tool for radiotherapy [1] or combined radiochemotherapy [2]. In this regard, the outcome of

\footnotetext{
* Correspondence: maximilian.niyazi@med.uni-muenchen.de

${ }^{1}$ Department of Radiation Oncology, University of Munich, Marchioninistr. 15, 81377, Munich, Germany

Full list of author information is available at the end of the article
}

head-and-neck cancer patients has been related to SUV changes in PET imaging [3]. Several tumour entities have been described in which data from PET-CT were predictive for pathological response, namely in rectal cancer $[4,5]$, NSCLC [6] or oesophageal cancer [7,8]. PET/CT was even described to be complimentarily to a CT scan and being able to predict early recurrence in breast cancer [9]. Recent trials on Hodgkin's disease are at least in part based on PET imaging and stratification is done according to PET results. Involved-node radiotherapy has been suggested as tool to further improve the therapeutic ratio by reducing radiation-induced toxicity [10].

\section{Biomed Central}

(c) 2013 Niyazi et al.; licensee BioMed Central Ltd. This is an Open Access article distributed under the terms of the Creative Commons Attribution License (http://creativecommons.org/licenses/by/2.0), which permits unrestricted use, distribution, and reproduction in any medium, provided the original work is properly cited. 
Altogether, PET is a relevant tool for a personalization in radiotherapy providing patho-anatomical information, predictive data, also information on prognosis and may be used for adaptive planning, e. g. in head-and-neck cancer with anatomical changes during therapy, in future [11]. Due to its ability to provide complementary information (tumour extent and physiology) to MRI and CT, $\mathrm{PET} / \mathrm{CT}$ has a potentially important place for radiotherapy planning $[12,13]$. In this regard, two aspects have to be distinguished: Firstly, PET imaging can be used for improved target volume delineation. Secondly, it may also be used for enhanced dose delivery to the target. For example, dose painting by contours (DPBC) consists of creating an additional PET-based target volume which will then be treated by a higher dose level [14-18]. In contrast, dose painting by numbers (DPBN) aims for a locally varying dose prescription according to the variation of the PET signal [19].

Many other tumour types are currently under investigation as PET provides additional information on the tumour extent, lymph node involvement and putative distant metastases $[20,21]$. Nevertheless, several problems have to be solved in future such as the inclusion of dynamic analyses or the correct thresholding procedures [22].

In order to compare manually delineated GTVs and automatically generated FDG-PET/CT based BTVs we retrospectively analysed a series of 20 consecutive patients who underwent planning PET/CT scanning at a dedicated $\mathrm{PET} / \mathrm{CT}$ scanner in treatment position. Tumour types were restricted to lung cancer, oesophageal cancer and pelvic cancer mainly consisting of cervical cancer with macroscopic tumor only. For this purpose, volumetric and geometric relationships between BTVs and GTVs were assessed.

\section{Methods \\ Patients}

20 consecutive patients received a planning PET/CT and were categorized into three groups: lung cancer, oesophageal cancer and solid cancer of the pelvic region. Scans were performed between 08/2010 and 09/ 2011 at the Department of Nuclear Medicine of the University of Munich. Only patients with a macroscopic primary tumour and unified GTVs were included into the retrospective analysis. Five of these patients received neoadjuvant chemotherapy upfront to radiotherapy, among these four lung and one oesophageal cancer patient (median duration from the last cycle to the PET scan was 2 weeks).

\section{$\left[{ }^{18} \mathrm{~F}\right]$-FDG PET and PET/CT}

Whole-body PET scans were acquired in three-dimensional (3D) mode extending from the proximal femora to the base of the skull or from the proximal femora including the skull (Biograph 64 TruePoint PET/CT, Siemens Medical Solutions or GE Discovery 690, GE Healthcare, Pollards Wood, United Kingdom).

After fasting for at least six hours, blood glucose levels were measured, a diuretic and an antispasmodic medication was administered intravenously, followed by bolus administration of $\left[{ }^{18} \mathrm{~F}\right]-\mathrm{FDG}$ (mean $228 \mathrm{MBq}$ ). Scans were performed with an emptied bladder. The emission sequence was initiated at approximately $60 \mathrm{~min}$ after intravenous injection of the $\left[{ }^{18} \mathrm{~F}\right]-\mathrm{FDG}$. Attenuation scanning was obtained by CT. Two nuclear medicine physicians performed a consensus evaluation of the final PET/CT or PET images using a dedicated software package (Hermes Hybrid Viewer Version 1.4, Hermes Medical Solution, Stockholm, Sweden).

\section{Definition and quantitative analysis of tumour volumes}

A consultant radiation oncologist without prior knowledge of the study delineated all GTVs. All the GTVs were mainly based on CT imaging and results of PET imaging were taken into account [23,24] without any systematic recommendation regarding thresholding or tracer amount (only the raw data as well as the report of the nuclear medicine expert were used as guidance for contouring within the axial CT slices); manually generated GTVs and their corresponding PTVs were used for treatment purpose in all patients included in this analysis.

A secondary automated lesion segmentation based on the given $\left[{ }^{18} \mathrm{~F}\right]$-FDG PET was performed using a dedicated software package (Hybrid Viewer, research version, Hermes Medical Solutions, Stockholm, Sweden) in two different ways: Based on maximal SUV ( $\mathrm{SUV}_{\max }$ ) thresholding and also according to the total lesion glycolysis (TLG) criteria described in PERCIST [25].

For the $\mathrm{SUV}_{\max }$ thresholding criteria, separate VOIs were generated for $38 \%, 42 \%, 47 \%$ and $50 \%$ of $\mathrm{SUV}_{\max }$ whereas these thresholds were chosen empirically based on literature, phantom studies and own experiences. The PERCIST TLG criteria were based on $\left[{ }^{18} \mathrm{~F}\right]$-FDG normal background activity determined from a $15 \mathrm{ml}$ VOI in the right hepatic lobe. The outer boundary of the lesion is equal to 3 standard deviations (SD) above normal-liver mean SUV. Subsequently all five generated VOIs were back-projected onto the original CT images used for therapy planning and exported as DICOM RT structure sets for comparison with the manually contoured GTV [25] (an example is shown in Figure 1).

To assess geometric differences between BTV and GTV the conformity index was computed (expressed as percentage). It is defined as the ratio between intersection and conjunction of both volumes. Additionally the ratio intersection between GTV/BTV and GTV volumes was calculated to provide an estimate of accordance with the manually delineated gold standard. 


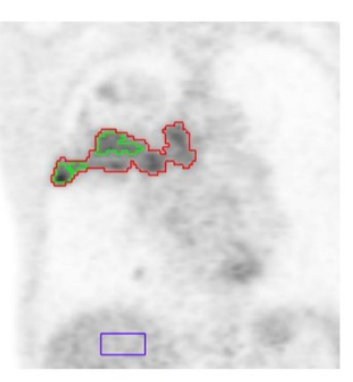

$50 \%$

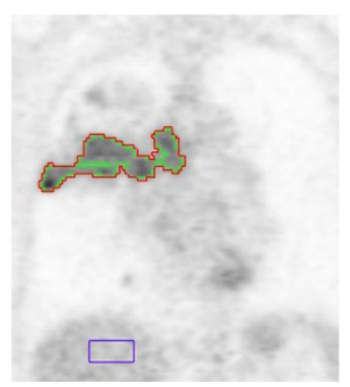

$42 \%$

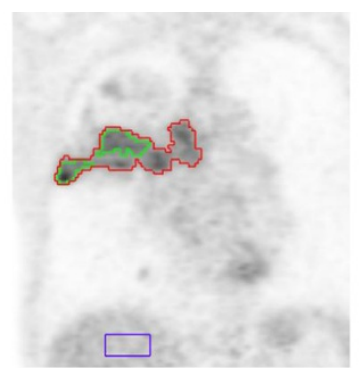

$47 \%$

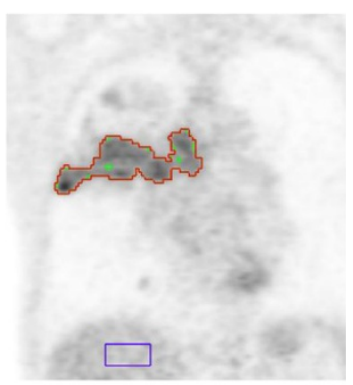

$38 \%$

red: PERCIST TLG violet: PERCIST TLG Background 30ml

Figure 1 The principle of thresholding is shown for one patient with a NSCLC; red for the PERCIST TLG algorithm, green for different percentage threshold algorithms.

\section{Statistical analysis}

We performed all analyses using the Statistical Package for Social Sciences (SPSS, Ver. 18.0, SPSS Inc, Chicago, IL). For descriptive analyses of patients' characteristics and volume sizes we used percentages and median scores. The GTV, and BTV delineation methods were compared using the Wilcoxon signed-rank test as numerical data were not normally distributed (paired tests); no corrections for multiple comparisons were performed. A two-tailed p-value of less or equal than 0.05 was considered to indicate statistical significance.

\section{Results}

Altogether, among the examined 20 patients, eight had lung cancer (40\%), five oesophageal (25\%) and seven (35\%) a cancer of the pelvic region (cervical/vulvar or rectal cancer). Thirteen patients were female (65\%) and median age was 66 years (range, 34-93 years), mean age $66 \pm 15$ years. Median SUV $_{\max }$ was 9.2 (3.1-18.2), for an overview see Table 1.

The PET/CT-based, manually contoured, GTV size was in median $43.7 \mathrm{ml}$. Concerning different thresholding algorithms, median volume of SUV (38\%) was $29.2 \mathrm{ml}$, median volume of SUV (42\%) $23.8 \mathrm{ml}$, of SUV (47\%) $19.3 \mathrm{ml}$ and of SUV (50\%) $14.5 \mathrm{ml}$; these values are also shown for the three defined tumour type subgroups (see Table 2).
Interestingly, concerning the volumes there were significant differences between the SUV percentage thresholds/region growing algorithm and the manually defined GTV in all cancer types. Non-significant results were seen for oesophageal cancer when comparing the PERCIST TLG result with the GTV defined by the radiation oncologist, for lung cancer (SUV (38\%), SUV (42\%)) and pelvic cancer (SUV (38\%), SUV (42\%), PERCIST TLG) (see pvalues in Table 2).

\section{Table 1 Patient characteristics}

\begin{tabular}{ll}
\hline Characteristic & Patients $(\mathbf{N}=\mathbf{2 0})$ \\
\hline Sex & \\
- Male & $7(35 \%)$ \\
- Female & $13(65 \%)$ \\
Median Age $[y r]$ & $66(34-93)$ \\
\hline Tumour type & \\
- NSCLC & $5(25 \%)$ \\
- SCLC & $3(15 \%)$ \\
- Cervical cancer & $4(20 \%)$ \\
- Oesophageal cancer & $5(25 \%)$ \\
- Vulvar cancer & $1(5 \%)$ \\
- Rectal cancer & $2(10 \%)$ \\
Median SUV & $9.2(3.1-18.2)$ \\
\hline
\end{tabular}


Table 2 Different thresholding volumes (median values), corresponding conformity indices and p-values of the comparison of these volumes to the GTV

\begin{tabular}{|c|c|c|c|c|c|c|c|c|c|c|c|c|}
\hline & $\begin{array}{l}\text { Whole } \\
\text { cohort }\end{array}$ & $\begin{array}{l}\text { p-value } \\
\text { (vs. GTV) }\end{array}$ & $\begin{array}{l}\text { Median Cl } \\
\text { (GTV) [\%] }\end{array}$ & Lung & $\begin{array}{l}\text { p-value } \\
\text { (vs. GTV) }\end{array}$ & $\begin{array}{l}\text { Median Cl } \\
\text { (GTV) [\%] }\end{array}$ & Oesophageal & $\begin{array}{l}\text { p-value } \\
\text { (vs. GTV) }\end{array}$ & $\begin{array}{l}\text { Median Cl } \\
\text { (GTV) [\%] }\end{array}$ & Pelvic & $\begin{array}{l}\text { p-value } \\
\text { (vs. GTV) }\end{array}$ & $\begin{array}{l}\text { Median Cl } \\
\text { (GTV) [\%] }\end{array}$ \\
\hline \multicolumn{13}{|l|}{ Manual } \\
\hline GTV [ml] & 43.7 & - & - & 81.1 & - & - & 34.2 & - & - & 70.4 & - & - \\
\hline $\begin{array}{l}\text { SUV(38\%) } \\
\text { [ml] }\end{array}$ & 29.2 & 0.04 & 32.9 & 30.9 & 0.33 & 24.0 & 15.2 & 0.04 & 51.9 & 28.7 & 0.24 & 29.8 \\
\hline $\begin{array}{l}\text { SUV(42\%) } \\
{[\mathrm{ml}]}\end{array}$ & 23.8 & 0.02 & 30.0 & 25.4 & 0.21 & 25.5 & 13.8 & 0.04 & 47.6 & 26.0 & 0.24 & 27.2 \\
\hline $\begin{array}{l}\text { SUV(47\%) } \\
{[\mathrm{ml}]}\end{array}$ & 19.3 & $<0.001$ & 37.8 & 17.1 & 0.04 & 24.2 & 12.0 & 0.04 & 41.8 & 22.5 & 0.03 & 39.4 \\
\hline $\begin{array}{l}\text { SUV(50\%) } \\
{[\mathrm{ml}]}\end{array}$ & 14.5 & $<0.001$ & 33.3 & 14.3 & 0.03 & 20.5 & 11.4 & 0.04 & 38.7 & 22.0 & 0.02 & 34.5 \\
\hline $\begin{array}{l}\text { PERCIST } \\
\text { TLG [ml] }\end{array}$ & 19.8 & 0.01 & 35.3 & 12.8 & 0.02 & 30.0 & 30.3 & 0.69 & 56.4 & 15.3 & 0.27 & 24.9 \\
\hline
\end{tabular}

In a further step, conformity indices as well as the ratio of algorithm-defined volume within the GTV were evaluated for the whole patient cohort.

The best agreement was determined with a fixed SUV (38\%) threshold: median $49 \%$ of this predefined volume was included within the radiation oncologist's GTV. The remaining results are also shown in Table 2. Using the PERCIST TLG algorithm 43.7\% were achieved.

Concerning conformity indices, the best agreement was determined with a fixed SUV(47\%) threshold: median $37.8 \%$ overlapped with the GTV. Using the PERCIST TLG algorithm, the median conformity index was $35.3 \%$. In a next step, the three subgroups defined by cancer site were analysed.

For lung cancer, the best agreement was determined by a fixed SUV (38\%) threshold: median $35.4 \%$ of this automatically generated volume was included within the radiation oncologist's GTV. Using the PERCIST TLG growing algorithm $30.2 \%$ of the respective BTV was included within the manually delineated GTV.

Contrarily, concerning conformity indices, the best agreement was determined with the PERCIST TLG algorithm: median $37.8 \%$ overlapped with the GTV.

For oesophageal cancer, the best agreement was found using the region-growing threshold: median $71.1 \%$ of this predefined volume was included within the radiation oncologist's GTV. Concerning conformity indices, the PERCIST TLG again determined the best agreement: median $56.4 \%$ overlap with the GTV.

For pelvic cancer, the best agreement was determined by the SUV (38\%) threshold: median $57.1 \%$ of this predefined volume was included within the radiation oncologist's GTV. Concerning conformity indices, the best agreement was determined with the SUV (47\%) algorithm: median $39.4 \%$ overlap with the GTV. For the remaining thresholds results were as follows: 29.8\% (SUV (38\%)),
27.2\% (SUV (42\%)), 34.5\% (SUV (50\%)) and 24.9\% (PERCIST TLG).

\section{Discussion}

Aim of this study was to examine in how far different PET segmentation algorithms have the potential to replace manually defined GTVs. Therefore, altogether 20 patients with cancer types of lung, esophagus or the pelvic region were considered, all of them bearing macroscopic tumours with no wide-spread disease.

Our data suggest that the general performance was rather limited with median conformity indices between $30.0-37.8 \%$. The best match with the GTV was obtained for the subgroup of oesophageal cancer patients with a conformity index of $56.4 \%$ and BTV within GTV ratio of $71.1 \%$ whereas potential explanations are reduced movement artifacts and smaller well-defined lesions compared to lung or pelvic tumours. Especially the performance of percentage thresholding algorithms was poor-partly as they are derived from phantom studies (and empirically from retrospective series) where a SUV of $40-50 \%$ of the maximum was suggested to be appropriate for GTV contouring of sphere tumours with homogeneously distributed $\left[{ }^{18} \mathrm{~F}\right]$-FDG [26]. However, these thresholds having been derived under exactly reproducible scientific conditions had no dependence on SUV variations and background/noise changes seen in clinical situations.

Regarding these three tumour types, many data are available and studies are ongoing. However, most of them have not included a dedicated planning PET/CT but a separate scan which bears the disadvantage of coregistration errors.

PET/CT has early been shown to improve accuracy of target volume delineation for treatment optimization in NSCLC [27], but taking a 40\% SUV $_{\max }$ threshold has been shown to be not appropriate for GTV definition 
[28]. Delineation studies exist showing that percentage volume changes from GTV [CT] to GTV [PET/CT] were lower for the nuclear medicine expert than for the radiation oncologists, suggesting a lower impact of PET/CT in target volume delineation for the nuclear medicine expert than for the radiation oncologists [29]; this observation is probably due to the radiation oncologist's experience on the natural tumour spread and thus a tendency to extend the respective GTV.

Besides an improved target volume definition, PET has been shown to reduce radiation treatment volumes caused by the avoidance of PET negative mediastinal lymph nodes in NSCLC patients. Thus it reduces toxicity with the same radiation dose or enables radiation dose escalation with the same toxicity. PET also reduces interobserver variability $[24,30]$ for delineating tumours and opens perspectives for more automated delineation parts in radiation treatment planning. Despite all inherent limitations present FDGPET/CT scans are frequently used in routine clinical practice for NSCLC [31,32].

Concerning the pelvic region, with a constantly advancing body of evidence and increasing availability, applications of PET/CT in colorectal and cervical cancer are likely to emerge in therapy response assessment [33], radiotherapy planning, use of novel tracers and one-stop-shop imaging techniques such as iodinated contrast-enhanced PET/CT [34,35].

In a recent study comparing MRI and PET/CT in cervical cancer patients it could be shown that the mean difference between MRI and PET volumes was least with $30 \% \mathrm{SUV}_{\max }$ threshold which is similar to our results [36].

Last but not least, when combined PET/CT was used for radiotherapy treatment planning in oesophageal cancer, there have been alterations to the delineation of tumour volumes when compared to CT alone, with the potential to avoid geographical misses of tumour [37]. This indicates a clinical role for FDG/PET in radiotherapy planning for oesophageal cancer [38].

Related to the retrospective nature of our study with a relatively small heterogeneous patient cohort several key limitations have to be stated.

Firstly, due to subjective adaptation of the manually derived GTVs (where inter- and intra-observer variability were not taken into account), these may be larger than the real tumour extent. Secondly, since in $5 / 20$ cases neoadjuvant chemotherapy was applied the given post-therapeutic tissue alterations may result in considerable problems for any auto-segmentation algorithm. Thirdly, co-registration was performed manually in a small number of cases leaving room for slightly imprecise correspondence of PET and CT information.

Furthermore, several minor problems have to be taken into account: Macroscopic tumour next to organs or structures with naturally higher glucose metabolism/uptake bears further problems for exact auto-segmentation as these structures have to be masked out manually. Additionally, distant secondary GTVs such as lymph node metastases may create problems related to their different FDG uptake pattern where a local background would be more suitable [39].

Thus, automated segmentation may indeed currently be used to complement the delineation process. But at present, none of the tested algorithms is able to fully replace the process of manual target volume definition and critical editing of any given PET signal.

From a radiation oncologist's point of view the most immanent need would be the development of robust algorithms for the reliable definition of macroscopic tumour extent or adequate processing constraints to generate CTV $[39,40]$ or PTV [41]. The most promising algorithms are background- and metabolically corrected algorithms such as the PERCIST TLG algorithm.

In the meantime, the use of PET for planning purposes requires a very close interaction of radiation oncologists, nuclear medicine experts and diagnostic radiologists for individual treatment planning. In this regard, several logistic issues have to be solved adequately: Who sets the contour at which time point, and how can all the available diagnostic information properly be integrated during the planning process?

\section{Conclusions}

All in all, there is only low concordance between manually derived GTVs and automatically segmented FDGPET/CT based BTVs at present indicating the need for further research in order to achieve higher volumetric conformity. Nevertheless, our data suggest that FDG-PET/CT may be regarded as an important tool for optimization of radiotherapy planning.

\section{Competing interests}

Andreas Elsner due to employment. The remaining authors declare that they have no competing interests.

\section{Authors' contributions}

UG, MH, AE, SL, FM, CB \& MN planned, coordinated and conducted the study. MN analysed the treatment planning data, fused imaging data set, performed associated statistics and wrote the manuscript. SL, AE \& MH performed PET imaging, provided the standard for PET-contouring, the fused data sets and contributed parts to the manuscript. All authors read, critically revised and approved the final manuscript.

\section{Author details}

'Department of Radiation Oncology, University of Munich, Marchioninistr. 15, 81377, Munich, Germany. ${ }^{2}$ Department of Nuclear Medicine, University of Munich, Marchioninistr. 15, 81377, Munich, Germany. ${ }^{3}$ Hermes Medical Solutions, Skeppsbron 44, 11130, Stockholm, Sweden.

Received: 30 April 2013 Accepted: 9 July 2013

Published: 12 July 2013

\section{References}

1. Parlak C, Topkan E, Onal C, Reyhan M, Selek U: Prognostic value of gross tumor volume delineated by FDG-PET-CT based radiotherapy treatment 
planning in patients with locally advanced pancreatic cancer treated with chemoradiotherapy. Radiat Oncol 2012, 7:37.

2. Bussink J, Kaanders JH, van der Graaf WT, Oyen WJ: PET-CT for radiotherapy treatment planning and response monitoring in solid tumors. Nat Rev Clin Oncol 2011, 8(4):233-242.

3. Allal AS, Slosman DO, Kebdani T, Allaoua M, Lehmann W, Dulguerov P: Prediction of outcome in head-and-neck cancer patients using the standardized uptake value of 2-[18F] fluoro-2-deoxy-D-glucose. Int $J$ Radiat Oncol Biol Phys 2004, 59(5):1295-1300.

4. de Geus-Oei L-F, Vriens D, van Laarhoven HWM, van der Graaf WTA, Oyen WJG: Monitoring and predicting response to therapy with (18)F-FDG PET in colorectal cancer: a systematic review. J NuCl Med 2009, 50:43S-54S.

5. Goldberg N, Kundel Y, Purim O, Bernstine H, Gordon N, Morgenstern S, Idelevich E, Wasserberg N, Sulkes A, Groshar D, et al: Early prediction of histopathological response of rectal tumors after one week of preoperative radiochemotherapy using 18 F-FDG PET-CT imaging. A prospective clinical study. Radiat Oncol 2012, 7:124

6. Pottgen C, Levegrun S, Theegarten D, Marnitz S, Grehl S, Pink R, Eberhardt W, Stamatis G, Gauler T, Antoch G, et al: Value of 18F-fluoro-2-deoxy-D-glucosepositron emission tomography/computed tomography in non-small-cell lung cancer for prediction of pathologic response and times to relapse after neoadjuvant chemoradiotherapy. Clin Cancer Res 2006, 12(1):97-106.

7. Wolf MC, Stahl M, Krause BJ, Bonavina L, Bruns C, Belka C, Zehentmayr F: Curative treatment of oesophageal carcinoma: current options and future developments. Radiat Oncol 2011, 6:55.

8. Song SY, Kim JH, Ryu JS, Lee GH, Kim SB, Park SI, Song HY, Cho KJ, Ahn SD, Lee SW, et al: FDG-PET in the prediction of pathologic response after neoadjuvant chemoradiotherapy in locally advanced, resectable esophageal cancer. Int J Radiat Oncol Biol Phys 2005, 63(4):1053-1059.

9. Evangelista L, Baretta Z, Vinante L, Cervino AR, Gregianin M, Ghiotto C, Saladini G, Sotti G: Tumour markers and FDG PET/CT for prediction of disease relapse in patients with breast cancer. Eur J Nucl Med Mol Imaging 2011, 38(2):293-301.

10. Kobe C, Dietlein M, Fuchs M: Interpretation and validation of interim positron emission tomography in Hodgkin lymphoma. Leuk Lymphoma 2010, 51(3):552-553.

11. Gregoire V, Jeraj R, Lee JA, O'Sullivan B: Radiotherapy for head and neck tumours in 2012 and beyond: conformal, tailored, and adaptive? Lancet Oncol 2012, 13(7):e292-e300.

12. Yaromina A, Zips D: Bio-IGRT. Biologically-adapted, forming truncated Radiotherapy. Nuklearmedizin 2010, 49(6A):S50-S52.

13. Daisne JF, Duprez T, Weynand B, Lonneux M, Hamoir M, Reychler H, Gregoire V: Tumor volume in pharyngolaryngeal squamous cell carcinoma: comparison at CT, MR imaging, and FDG PET and validation with surgical specimen. Radiology 2004, 233(1):93-100.

14. Piroth MD, Pinkawa M, Holy R, Klotz J, Schaar S, Stoffels G, Galldiks N, Coenen $\mathrm{HH}$, Kaiser HJ, Langen $\mathrm{KJ}$, et al: Integrated boost IMRT with FETPET-adapted local dose escalation in glioblastomas. Results of a prospective phase II study. Strahlenther Onkol 2012, 188(4):334-339.

15. Fodor A, Fiorino C, Dell'Oca I, Broggi S, Pasetti M, Cattaneo GM, Gianolli L, Calandrino R, Di Muzio NG: PET-guided dose escalation tomotherapy in malignant pleural mesothelioma. Strahlenther Onkol 2011, 187(11):736-743.

16. Pinkawa M, Piroth MD, Holy R, Klotz J, Djukic V, Corral NE, Caffaro M, Winz $\mathrm{OH}$, Krohn T, Mottaghy FM, et al: Dose-escalation using intensitymodulated radiotherapy for prostate cancer-evaluation of quality of life with and without (18)F-choline PET-CT detected simultaneous integrated boost. Radiat Oncol 2012, 7:14.

17. Niyazi M, Bartenstein P, Belka C, Ganswindt U: Choline PET based dosepainting in prostate cancer-modelling of dose effects. Radiat Oncol 2010, 5:23.

18. Wurschmidt F, Petersen C, Wahl A, Dahle J, Kretschmer M: [18F] fluoroethylcholine-PET/CT imaging for radiation treatment planning of recurrent and primary prostate cancer with dose escalation to PET/CTpositive lymph nodes. Radiat Oncol 2011, 6:44.

19. Thorwarth D, Geets X, Paiusco M: Physical radiotherapy treatment planning based on functional PET/CT data. Radiother Oncol 2010, 96(3):317-324

20. Schober O: PET/CT in head and neck squamous cell carcinoma with an unknown primary: significant diagnostic benefit in a prospective clinical trial. Strahlenther Onkol 2012, 188(7):637-638.
21. MacDonald SL, Mulroy L, Wilke DR, Burrell S: PET/CT aids the staging of and radiotherapy planning for early-stage extranodal natural killer/T-cell lymphoma, nasal type: a case series. Radiat Oncol 2012, 6:182.

22. Cheebsumon $P$, Yaqub $M$, van Velden FH, Hoekstra OS, Lammertsma AA, Boellaard R: Impact of [(1)(8)F]FDG PET imaging parameters on automatic tumour delineation: need for improved tumour delineation methodology. Eur J Nucl Med Mol Imaging 2011, 38(12):2136-2144.

23. Nestle U, Walter K, Schmidt S, Licht N, Nieder C, Motaref B, Hellwig D, Niewald M, Ukena D, Kirsch CM, et al: 18F-deoxyglucose positron emission tomography (FDG-PET) for the planning of radiotherapy in lung cancer: high impact in patients with atelectasis. Int J Radiat Oncol Biol Phys 1999 44(3):593-597.

24. Ciernik IF, Dizendorf E, Baumert BG, Reiner B, Burger C, Davis JB, Lutolf UM, Steinert HC, Von Schulthess GK: Radiation treatment planning with an integrated positron emission and computer tomography (PET/CT): a feasibility study. Int J Radiat Oncol Biol Phys 2003, 57(3):853-863.

25. Wahl RL, Jacene $H$, Kasamon $Y$, Lodge MA: From RECIST to PERCIST: evolving considerations for PET response criteria in solid tumors. $J$ NuCl Med 2009, 50(Suppl 1):122S-150S.

26. Uto F, Shiba E, Onoue S, Yoshimura H, Takada M, Tsuji Y, Fukugami S, Asakawa I, Tamamoto T, Hasegawa M: Phantom study on radiotherapy planning using PET/CT-delineation of GTV by evaluating SUV. J Radiat Res 2010, 51(2):157-164.

27. Greco C, Rosenzweig K, Cascini GL, Tamburrini O: Current status of PET/CT for tumour volume definition in radiotherapy treatment planning for non-small cell lung cancer (NSCLC). Lung Cancer 2007, 57(2):125-134.

28. Nestle U, Kremp S, Schaefer-Schuler A, Sebastian-Welsch C, Hellwig D, Rube C, Kirsch CM: Comparison of different methods for delineation of 18FFDG PET-positive tissue for target volume definition in radiotherapy of patients with non-small cell lung cancer. J Nucl Med 2005, 46(8):1342-1348.

29. Hanna GG, Carson KJ, Lynch T, McAleese J, Cosgrove VP, Eakin RL, Stewart DP, Zatari A, O'Sullivan JM, Hounsell AR: 18F-fluorodeoxyglucose positron emission tomography/computed tomography-based radiotherapy target volume definition in non-small-cell lung cancer: delineation by radiation oncologists vs. joint outlining with a PET radiologist? Int J Radiat Oncol Biol Phys 2010, 78(4):1040-1051.

30. Vees H, Casanova N, Zilli T, Imperiano H, Ratib O, Popowski Y, Wang H, Zaidi H, Miralbell R: Impact of 18F-FDG PET/CT on target volume delineation in recurrent or residual gynaecologic carcinoma. Radiat Oncol 2012, 7:176.

31. De Ruysscher D: PET-CT in radiotherapy for lung cancer. Positron Emission Tomography 2011, 727:53-58

32. De Ruysscher $D$, Nestle $U$, Jeraj $R$, Macmanus M: PET scans in radiotherapy planning of lung cancer. Lung Cancer 2012, 75(2):141-145.

33. Brooks FJ, Grigsby PW: Current measures of metabolic heterogeneity within cervical cancer do not predict disease outcome. Radiat Oncol 2011, 6:69

34. Chowdhury FU, Shah N, Scarsbrook AF, Bradley KM: (18)F FDG PET/CT imaging of colorectal cancer: a pictorial review. Postgrad Med J 2010, 86(1013):174-182.

35. Salem A, Salem AF, Al-Ibraheem A, Lataifeh I, Almousa A, Jaradat I: Evidence for the use PET for radiation therapy planning in patients with cervical cancer: a systematic review. Hematol Oncol Stem Cell Ther 2011, 4(4):173-181.

36. Upasani MN, Mahantshetty UM, Rangarajan V, Purandare N, Merchant N, Thakur M, Engineer R, Chopra S, Shrivastava SK: 18-fluoro-deoxy-glucose positron emission tomography with computed tomography-based gross tumor volume estimation and validation with magnetic resonance imaging for locally advanced cervical cancers. Int J Gynecol Cancer 2012, 22(6):1031-1036

37. Leong T, Everitt $C$, Yuen $K$, Condron S, Hui A, Ngan SY, Pitman A, Lau EW, MacManus M, Binns D, et al: A prospective study to evaluate the impact of FDG-PET on CT-based radiotherapy treatment planning for oesophageal cancer. Radiother Oncol 2006, 78(3):254-261.

38. Vrieze O, Haustermans K, De Wever W, Lerut T, Van Cutsem E, Ectors N, Hiele M, Flamen P: Is there a role for FGD-PET in radiotherapy planning in esophageal carcinoma? Radiother Oncol 2004, 73(3):269-275.

39. Ciernik IF, Brown DW, Schmid D, Hany T, Egli P, Davis JB: 3D-segmentation of the 18F-choline PET signal for target volume definition in radiation therapy of the prostate. Technol Cancer Res Treat 2007, 6(1):23-30. 
40. Messa C, Ceresoli GL, Rizzo G, Artioli D, Cattaneo M, Castellone P, Gregorc V, Picchio M, Landoni C, Fazio F: Feasibility of [18F]FDG-PET and coregistered $\mathrm{CT}$ on clinical target volume definition of advanced nonsmall cell lung cancer. Q J Nucl Med Mol Imaging 2005, 49(3):259-266.

41. Ciernik IF, Huser M, Burger C, Davis JB, Szekely G: Automated functional image-guided radiation treatment planning for rectal cancer. Int $J$ Radiat Oncol Biol Phys 2005, 62(3):893-900.

doi:10.1186/1748-717X-8-180

Cite this article as: Niyazi et al:: Automated biological target volume

delineation for radiotherapy treatment planning using

FDG-PET/CT. Radiation Oncology 2013 8:180.

\section{Submit your next manuscript to BioMed Central and take full advantage of:}

- Convenient online submission

- Thorough peer review

- No space constraints or color figure charges

- Immediate publication on acceptance

- Inclusion in PubMed, CAS, Scopus and Google Scholar

- Research which is freely available for redistribution 